\title{
CARACTERÍSTICAS DA ESPECTROGRAFIA DE BANDA LARGA E ESTREITA DA EMISSÃO VOCAL DE HOMENS COM LARINGE SEM AFECÇÕES
}

\section{Features of wide and narrow band spectrography as for vocal emission of men with larynx without diseases}

\author{
Bárbara Costa Beber ${ }^{(1)}$, Carla Aparecida Cielo (2)
}

\begin{abstract}
RESUMO
Objetivo: caracterizar as espectrografias vocais de um grupo de homens adultos jovens. Método: 25 homens adultos jovens, sem queixas vocais, com diagnóstico otorrinolaringológico de laringe sem afecção, realizaram a emissão sustentada da vogal [a] em pé e com frequência e intensidade habituais. As emissões foram analisadas pelo programa Real Time Spectrogram da Kay Pentax ${ }^{\circledR}$, que originou um espectrograma de banda larga e outro de banda estreita para cada sujeito. Estes foram duplicados, para aumentar a fidedignidade do estudo, e enviados a três fonoaudiólogas juízas que realizaram o julgamento dos mesmos seguindo um protocolo. Os resultados foram analisados estatisticamente pelo Teste para Diferenças de Proporções com nível de significância de $5 \%$. Resultados: na Espectrografia de Banda Larga, houve significância estatística para ruído muito presente nas frequências em torno de $3,2 \mathrm{KHz}$, pouca definição do terceiro formante, pouca regularidade do traçado e anti-ressonância mediana. Na Espectrografia de Banda Estreita, houve fraca intensidade das frequências em torno de $3,2 \mathrm{KHz}$ e em todo espectro, ruído muito presente em todo espectro e nas frequências em torno de $3,2 \mathrm{KHz}$, ruído mediano nas baixas frequências, pouca regularidade do traçado e anti-ressonância mediana. Conclusão: a análise espectrográfica dos homens adultos jovens, com laringe sem afecções e sem queixas vocais, revelou grande quantidade de ruído em todo o espectro e nas frequências em torno de $3,2 \mathrm{KHz}$, terceiro formante pouco definido, ruído mediano nas baixas frequências, traçado pouco regular, anti-ressonância mediana, fraca intensidade em todo espectro e, em especial, nas frequências em torno de $3,2 \mathrm{KHz}$.
\end{abstract}

DESCRITORES: Voz; Saúde do Homem; Espectrografia; Fonoaudiologia; Laringe

\section{INTRODUÇÃO}

Os dados espectrográficos vocais podem ser representados por um gráfico tridimensional que apresenta em sua ordenada a frequência, em sua

(1) Fonoaudióloga efetiva da Prefeitura Municipal de Mostardas, Palmares do Sul, Rio Grande do Sul, Brasil; Mestre em Distúrbios da Comunicação Humana pela Universidade Federal de Santa Maria, Rio Grande do Su.

(2) Fonoaudióloga; Professora Adjunta do Curso de Fonoaudiologia e do Programa de Pós-Graduação em Distúrbios da Comunicação Humana da Universidade Federal de Santa Maria, Rio Grande do Sul, Brasil; Doutora em Linguística Aplicada pela Pontifícia Universidade Católica do Rio Grande do Sul.

Conflito de interesses: inexistente abscissa o tempo, e seu grau de escurecimento do traçado relaciona-se com a intensidade. A espectrografia reflete dados relativos à fonte glótica e dados da postura do trato vocal, caracterizando vogais e consoantes ${ }^{1-4}$ e fornecendo dados referentes à distribuição dos harmônicos e dos formantes $(F)$ do som glótico'.

Fatores como a idade, a raça e o sexo são determinantes das dimensões e da fisiologia do trato vocal, consequentemente, os resultados da análise espectrográfica são influenciados por esses fatores ${ }^{4-8}$.

Existem poucos estudos caracterizando especificamente vozes masculinas normais. Os trabaIhos que trazem informações acerca do assunto 
relatam que as vozes masculinas são mais graves, apresentam formantes deslocados para a parte inferior do espectro, possuem harmônicos mais graves e menos lineares, apresentam imagem espectrográfica com escurecimento mais variável e menos regular e, ainda, podem ser mais ruidosas e apresentarem medidas elevadas de jitter e shimmer $2-5,9,10$.

É importante que existam estudos sobre características vocais que forneçam dados norteadores sobre grupos com voz normal, pois poderão servir à área de voz profissional para a avaliação e comparação de resultados iniciais e de metas atingidas pelo trabalho de aperfeiçoamento vocal fonoaudiológico.

Com base no exposto, esta pesquisa objetivou caracterizar as espectrografias vocais de um grupo de homens adultos jovens.

\section{MÉTODO}

Este estudo é uma análise qualitativa, transversal e exploratória, realizada por meio do levantamento de dados em banco de dados (primeira etapa) e em campo (segunda etapa).

Os dados da primeira etapa, que estavam armazenados em um banco de dados no Laboratório de Voz da instituição de origem, até o momento não haviam sido utilizados em outras pesquisas. Como os sujeitos do banco de dados já haviam assinado o Termo de Consentimento Livre e Esclarecido (TCLE) da instituição, não houve necessidade de termo próprio para essa etapa. Já a segunda etapa da pesquisa, teve início após leitura e assinatura do TCLE específico da pesquisa, conforme a Resolução 196/96, da Comissão Nacional de Ética em Pesquisa (CONEP), por todos os sujeitos da pesquisa.

Salienta-se que, no Laboratório de Voz, há procedimentos e protocolos-padrão para as avaliações em que todos os indivíduos passam pelos mesmos passos e procedimentos para uniformização dos dados. Desta forma, todos os sujeitos do banco de dados passaram, além das avaliações fonoaudiológicas, por exame médico otorrinolaringológico, triagem auditiva e coleta de emissões vocais nas mesmas condições do grupo de campo.

O grupo de estudo, foi constituído de forma intencional, por meio de critérios de inclusão e de exclusão. Os critérios de inclusão foram: ser do sexo masculino, apresentar diagnóstico otorrinolaringológico de laringe sem afecções, faixa etária de adulto jovem (20 a 40anos) e aderir ao TCLE.

Os critérios de exclusão foram: antecedentes médicos de doenças neurológicas, psiquiátricas, endocrinológicas ou gástricas; queixas vocais (rouquidão, fadiga vocal, falhas na voz ou ardência); diagnóstico otorrinolaringológico de afecções laríngeas; gripe, alergias respiratórias ou outras doenças que pudessem limitar o desempenho da produção vocal; hábitos de etilismo e tabagismo; tratamento fonoaudiológico e/ou otorrinolaringológico prévios; exame audiológico, evidenciando alterações auditivas; alterações do sistema estomatognático; ser cantor (a); idade abaixo de 20 anos; idade acima de 40 anos.

$\mathrm{Na}$ primeira etapa (13 sujeitos), os sujeitos do banco de dados foram selecionados a partir da análise das avaliações armazenadas, visando aos critérios de inclusão e de exclusão.

Na segunda etapa (12 sujeitos), após os voluntários assinarem ao TCLE, responderem a um questionário, realizarem avaliação otorrinolaringológica e triagem fonoaudiológica, que incluiu avaliação miofuncional orofacial e triagem auditiva, foram selecionados para as avaliações os sujeitos que se enquadravam nos critérios de inclusão e de exclusão.

Na triagem auditiva, optou-se por uma varredura de tons puros nas frequências de 500, 1000, 2000 e $4000 \mathrm{~Hz}$ a $25 \mathrm{~dB}$, somente pela via aérea ${ }^{11}$.

Foram selecionados 25 homens adultos jovens (13 selecionados na primeira etapa e 12 na segunda etapa), com idades entre 20 e 39 anos (média de 28 anos).

A coleta das vozes a serem analisadas já havia sido realizada anteriormente pelos sujeitos da primeira etapa e foi realizada da mesma maneira pelos sujeitos da segunda etapa. A emissão sustentada da vogal [a] foi coletada, solicitando-se ao sujeito que ficasse em pé, com os braços estendidos ao longo do corpo. O microfone acoplado ao gravador digital da marca Sony, modelo ICD-P210, foi posicionado em ângulo de $90^{\circ}$ graus e a $4 \mathrm{~cm}$ da boca b $^{1,312}$.

A emissão foi sustentada, em frequência e intensidade habituais, após inspiração profunda, emitindo o som em tempo máximo de fonação $(\mathrm{TMF})^{3}$.

Para a análise espectrográfica da voz, excluiuse o ataque vocal e a partir disso foram extraídos os 3,5 segundos iniciais da emissão da vogal [a] para a análise $e^{1,3,13}$.

Todas as emissões foram submetidas ao Programa Real Time Spectrogram da Kay Pentax ${ }^{\circledR}$, instalado em computador adequado a todas as especificações do fabricante, em filtro de Banda Larga 100 points $(646.00 \mathrm{~Hz})$ e em Filtro de Banda Estreita 1024 points (63.09 HZ), com amostra de $11 \mathrm{KHz}$ e 16 bits de quantização para análise espectrográfica das vozes na resolução de $3.200 \mathrm{~Hz}$ para a análise de vogais sustentadas consideradas 
normais, em que pode ocorrer um forte pico espectral entre 2500 e $3500 \mathrm{~Hz}$, correspondendo ao agrupamento de F3 e F4. Além disso, vozes de falantes normais, que não possuem treino vocal, não costumam apresentar boa definição dos formantes superiores, sendo que F4 e F5 podem não ser observáveis ${ }^{6,14-16}$.

Para a caracterização da Espectrografia de Banda Larga (EBL), foram avaliados os seguintes aspectos: Intensidade dos Formantes (F1, F2, F3); Intensidade do traçado nas frequências em torno de $3,2 \mathrm{KHz}$; Intensidade em todo o Espectro Vocal; Presença de Ruído; Largura de Banda dos Formantes (F1, F2, F3); Definição dos Formantes (F1, F2, F3); Regularidade do Traçado e Anti-Ressonância ${ }^{12,17-19}$

$\mathrm{Na}$ Espectrografia de Banda Estreita (EBE), foram considerados: Intensidade do traçado nas frequências em torno de $3,2 \mathrm{KHz}$ e Intensidade em todo o Espectro Vocal; Presença de Ruído; Definição de Harmônicos; Regularidade do Traçado e Anti-ressonância ${ }^{12,17-19}$

Quanto à intensidade (dos formantes, do traçado em frequências em torno de $3,2 \mathrm{KHz}$ e em todo o espectro), as juízas deviam considerar o grau de escurecimento do espectrograma que variava de preto (forte intensidade), cinza (intensidade mediana) a cinza claro (fraca intensidade $)^{17}$.

A presença de ruído foi caracterizada por uma imagem sombreada ou pontilhada no espectrograma, podendo estar ausente ou presente ${ }^{17,19} \mathrm{em}$ pouca, muita ou quantidade mediana, conforme o grau em cor preta do sombreado/pontilhado.

Os formantes foram classificados conforme a largura de sua banda em: pouca largura de banda (formante estreito), muita largura de banda (formante largo e amplo) e largura de banda mediana (formante nem largo nem estreito).

Os formantes e harmônicos visíveis, bem demarcados e simétricos foram considerados muito definidos; com pouca definição, foram considerados aqueles pouco visíveis, pouco demarcados e assimétricos; formantes e harmônicos com definição mediana situaram-se entre os dois anteriores.

A regularidade do traçado está relacionada à sua continuidade e estabilidade e foi classificada em muito regular, quando contínuo, sem falhas, interrupções ou oscilações; pouco regular, quando o traçado foi descontínuo, falhado, interrompido e ou com presença de oscilações (irregular); a regularidade mediana ficou entre ambos ${ }^{17}$.

A anti-ressonância (ou efeito damping) é descrita na literatura como um abafamento acústico decorrente do amortecimento do som, podendo estar relacionada com o direcionamento do som para a cavidade nasal. Também aparece descrita na literatura como: interrupção do formante, denso aglomerado de ressonâncias (cluster), bifurcação do formante, junção do formante, queda de intensidade do formante e presença de formantes nasais entre formantes orais ${ }^{1-3,20}$. Na classificação deste parâmetro, no presente estudo, quando se observou anti-ressonância muito visível e aparente, ela foi classificada como muito presente. Quando não foi observada anti-ressonância ou quando houve dúvida da sua presença por apresentar-se muito sutil, classificou-se como pouco presente. Quando sua presença ficou entre essas duas opções, classificou-se como mediana.

Esta análise foi realizada individualmente por três fonoaudiólogas, com experiência e mestrado na área de voz, considerando-se os dados marcados em comum ou predominantes nos julgamentos das avaliadoras. As espectrografias foram duplicadas, a fim de aumentar a fidedignidade da avaliação de cada juíza, sem que as mesmas soubessem.

As espectrografias foram duplicadas, a fim de aumentar a fidedignidade da avaliação de cada juíza, sem que as mesmas soubessem. Assim, após o julgamento das espectrografias pelas juízas, foram considerados os dados marcados em comum ou predominantes entre os julgamentos das avaliadoras.

Cada um dos 25 sujeitos do grupo de estudos possuía duas espectrografias (uma de banda larga e uma de banda estreita), totalizando 50 gráficos; como esses gráficos foram duplicados (100 espectrografias) e como essas 100 espectrografias foram avaliadas por três juízas, originaram-se 300 julgamentos.

O estudo foi aprovado pelo Comitê de Ética em Pesquisa (CEP) da instituição de origem sob número 0087.0.243.000-07.

Após, os dados foram tabulados e analisados estatisticamente pelo teste para Diferença de Proporções em nível de significância de 5\%. Com o objetivo de caracterizar as espectrografias das vozes do grupo de estudo a partir do maior percentual de julgamentos em cada aspecto analisado, realizou-se um cruzamento do percentual das opções de resposta entre si. Para afirmar que o maior percentual de respostas marcada pelas juízas foi significativo em relação às outras opções, foi necessário que dois dos três cruzamentos em cada aspecto analisado apontassem significância estatística.

\section{RESULTADOS}

As tabelas apresentam os resultados estatísticos dos cruzamentos das opções de resposta da EBL (Tabela 1) e da EBE (Tabela 2). 
Tabela 1 - Resultados estatísticos da EBL de homens com voz e laringe normais

\begin{tabular}{|c|c|c|c|c|c|c|c|c|}
\hline \multirow{2}{*}{ Banda Larga } & \multicolumn{2}{|c|}{ Fraca } & \multicolumn{2}{|c|}{ Forte } & \multicolumn{2}{|c|}{ Mediana } & \multirow{2}{*}{ Total } & \multirow{2}{*}{ p (FRxF; FxM; FRxM) } \\
\hline & $\mathbf{n}$ & $\%$ & $\mathbf{n}$ & $\%$ & $\mathbf{n}$ & $\%$ & & \\
\hline Intensidade do F1 & 25 & $17 \%$ & 72 & $48 \%$ & 53 & $35 \%$ & 150 & $0,0120^{*} ; 0,2180 ; 0,1550$ \\
\hline Intensidade do F2 & 16 & $11 \%$ & 79 & $53 \%$ & 55 & $37 \%$ & 150 & $0,0050^{*} ; 0,0990 ; 0,0940$ \\
\hline Intensidade do F3 & 56 & $37 \%$ & 42 & $28 \%$ & 52 & $35 \%$ & 150 & 0,$4510 ; 0,6400 ; 0,9300$ \\
\hline Intensidade do traçado nas frequências em torno de $3,2 \mathrm{KHz}$ & 64 & $43 \%$ & 22 & $15 \%$ & 64 & $43 \%$ & 150 & 0,$8030 ; 0,0350 * ; 0,8580$ \\
\hline \multirow[t]{3}{*}{ Intensidade em todo espectro vocal } & 41 & $27 \%$ & 45 & $30 \%$ & 64 & $43 \%$ & 150 & 0,$9730 ; 0,2530 ; 0,1670$ \\
\hline & \multicolumn{2}{|c|}{ Pouca } & \multicolumn{2}{|c|}{ Muita } & \multicolumn{2}{|c|}{ Mediana } & \multirow[t]{2}{*}{ Total } & \multirow{2}{*}{ p (PxMT; MTxMD; PxMD) } \\
\hline & $\mathbf{n}$ & $\%$ & $\mathbf{n}$ & $\%$ & $\mathbf{n}$ & $\%$ & & \\
\hline Presença de ruído em todo espectro & 3 & $2 \%$ & 105 & $70 \%$ & 42 & $28 \%$ & 150 & 0,$0620 ; 0,0000^{*} ; 0,7050$ \\
\hline Presença de ruído nas frequências em torno de $3,2 \mathrm{KHz}$ & 3 & $2 \%$ & 110 & $73 \%$ & 37 & $25 \%$ & 150 & $0,0400^{*} ; 0,0000^{*} ; 0,7980$ \\
\hline Presença de ruído nas médias frequências & 3 & $2 \%$ & 82 & $55 \%$ & 65 & $43 \%$ & 150 & 0,$2280 ; 0,2280 ; 0,2280$ \\
\hline Presença de ruído nas baixas frequências & 3 & $2 \%$ & 45 & $30 \%$ & 102 & $68 \%$ & 150 & 0,$7030 ; 0,0000 * ; 0,0700$ \\
\hline Largura de banda do F1 & 39 & $26 \%$ & 54 & $36 \%$ & 57 & $38 \%$ & 150 & 0,4260; 0,9030; 0,3140 \\
\hline Largura de banda do F2 & 30 & $20 \%$ & 56 & $37 \%$ & 64 & $43 \%$ & 150 & 0,$1590 ; 0,6840 ; 0,0560$ \\
\hline Largura de banda do F3 & 48 & $32 \%$ & 31 & $21 \%$ & 71 & $47 \%$ & 150 & 0,$3990 ; 0,0210^{*} ; 0,1400$ \\
\hline Definição do F1 & 63 & $42 \%$ & 38 & $25 \%$ & 49 & $33 \%$ & 150 & 0,$1390 ; 0,4150 ; 0,6120$ \\
\hline Definição do F2 & 57 & $38 \%$ & 38 & $25 \%$ & 55 & $37 \%$ & 150 & 0,$2870 ; 0,3550 ; 0,9610$ \\
\hline Definição do F3 & 91 & $61 \%$ & 12 & $8 \%$ & 47 & $31 \%$ & 150 & $0,0020^{\star} ; 0,2050 ; 0,0031^{*}$ \\
\hline Regularidade do traçado & 92 & $61 \%$ & 15 & $10 \%$ & 43 & $29 \%$ & 150 & $0,0000^{*} ; 0,0000^{*} ; 0,2680$ \\
\hline Anti-ressonância-Damping em todo espectro & 3 & $2 \%$ & 37 & $25 \%$ & 110 & $73 \%$ & 150 & 0,$8540 ; 0,0000^{*} ; 0,0400^{\star}$ \\
\hline
\end{tabular}

Tabela 2 - Resultados estatísticos da EBE de homens com voz e laringe normais

\begin{tabular}{|c|c|c|c|c|c|c|c|c|}
\hline \multirow{2}{*}{ Banda Estreita } & \multicolumn{2}{|c|}{ Fraca } & \multicolumn{2}{|c|}{ Forte } & \multicolumn{2}{|c|}{ Mediana } & \multirow{2}{*}{ Total } & \multirow[t]{2}{*}{ p (FRxF; FxM; FRxM) } \\
\hline & $\mathbf{n}$ & $\%$ & $\mathbf{n}$ & $\%$ & $\mathbf{n}$ & $\%$ & & \\
\hline Intensidade do traçado nas frequências em torno de $3,2 \mathrm{KHz}$ & 93 & $62 \%$ & 10 & $7 \%$ & 47 & $31 \%$ & 150 & $0,0030^{*} ; 0,2290 ; 0,0001^{*}$ \\
\hline \multirow[t]{3}{*}{ Intensidade em todo espectro vocal } & 69 & $46 \%$ & 15 & $10 \%$ & 66 & $44 \%$ & 150 & $0,0220^{*} ; 0,9520 ; 0,0031^{*}$ \\
\hline & \multicolumn{2}{|c|}{ Pouca } & \multicolumn{2}{|c|}{ Muita } & \multicolumn{2}{|c|}{ Mediana } & \multirow[t]{2}{*}{ Total } & \multirow[t]{2}{*}{ p (PxMT; MTxMD; PxMD) } \\
\hline & $\mathbf{n}$ & $\%$ & $\mathbf{n}$ & $\%$ & $\mathbf{n}$ & $\%$ & & \\
\hline Presença de ruído em todo espectro & 9 & $6 \%$ & 110 & $73 \%$ & 31 & $21 \%$ & 150 & $0,0000^{*} ; 0,0000^{*} ; 0,6010$ \\
\hline Presença de ruído nas frequências em torno de $3,2 \mathrm{KHz}$ & 10 & $7 \%$ & 108 & $72 \%$ & 32 & $21 \%$ & 150 & $0,0000^{*} ; 0,0000^{*} ; 0,5590$ \\
\hline Presença de ruído nas médias frequências & 4 & $3 \%$ & 94 & $63 \%$ & 52 & $35 \%$ & 150 & 0,$0600 ; 0,0020^{*} ; 0,4450$ \\
\hline Presença de ruído nas baixas frequências & 9 & $6 \%$ & 49 & $33 \%$ & 92 & $61 \%$ & 150 & 0,$2200 ; 0,0002^{*} ; 0,0040^{*}$ \\
\hline Definição de harmônicos & 60 & $40 \%$ & 51 & $34 \%$ & 39 & $26 \%$ & 150 & 0,$6490 ; 0,5580 ; 0,2240$ \\
\hline Regularidade do traçado & 103 & $69 \%$ & 21 & $14 \%$ & 26 & $17 \%$ & 150 & $0,0000^{*} ; 0,9280 ; 0,0000^{*}$ \\
\hline Anti-ressonância/ damping em todo espectro & 3 & $2 \%$ & 21 & $14 \%$ & 126 & $84 \%$ & 150 & 0,$7300 ; 0,0000^{*} ; 0,0040^{*}$ \\
\hline
\end{tabular}

\section{DISCUSSÃO}

A literatura relata que os formantes inferiores, Primeiro Formante (F1) e Segundo Formante (F2), relacionam-se com a altura e anterioridade da língua dentro da cavidade oral, sendo capaz de determinar a qualidade da vogal, dando identidade às mesmas, enquanto o Terceiro Formante (F3), Quarto Formante (F4) e Quinto Formante (F5) relacionam-se com a qualidade vocal. Isso acontece porque as frequências dos formantes inferiores estão relacionadas à configuração da língua no trato vocal e os formantes superiores ao comprimento do trato vocal e a dimensão dentro e em torno da laringe. Os formantes superiores são mais evidentes em vozes de cantores e atores, pois suas vozes apresentam melhor qualidade e projeção. Portanto, vozes de falantes normais, que não 
possuem treino vocal, não costumam apresentar boa definição dos formantes superiores, sendo que

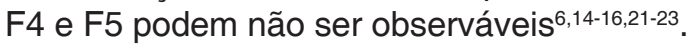

No presente estudo, a EBL apresentou pouca definição de F3, com significância estatística, mostrando estar de acordo com a literatura ${ }^{6,14-16,21-25}$ (Tabela 1). No entanto, é possível que a amostragem de $3,2 \mathrm{KHz}$ tenha comprometido a qualidade de visualização de F3 pelo fato do mesmo se encontrar no limite da amostragem.

$\mathrm{Na}$ EBE, a intensidade em todo espectro e especificamente nas frequências em torno de $3,2 \mathrm{KHz}$, mostrou-se significantemente fraca (Tabela 1). $\mathrm{Na}$ espectrografia, a intensidade é determinada pelo grau de escurecimento do traçado, que se relaciona com a loudness e pode estar alterado em vozes soprosas, astênicas e tensas ${ }^{24,25}$. A falta de energia dos harmônicos tem relação com a falta de energia na vocalização ou impossibilidade anatômica de fazê-lo ${ }^{26}$. Vozes disfônicas costumam apresentar harmônicos fracos, características como harmônicos, pouco definidos e irregulares ${ }^{1,3}$. Como os indivíduos do grupo estudado não tinham vozes treinadas, eram esperadas algumas irregularidades, decorrentes de fatores como a incoordenação pneumofonoarticulatória, por uma relação desarmônica entre as forças mioelásticas da laringe e expiratórias dos pulmões. A apresentação dos harmônicos se refere também à projeção e ressonância vocal ${ }^{4}$. Portanto, a fraca intensidade também pode ser uma característica da falta de projeção das vozes não treinadas ${ }^{27}$.

Tanto na EBL como na EBE, o ruído esteve significantemente muito presente no traçado das frequências em torno de $3,2 \mathrm{KHz}$ (Tabelas 1 e 2).

A presença de ruído nessa região é compatível com uma espectrografia alterada, sugerindo rouquidão ou soprosidade 1,3,24-26,28-30. Esse achado concorda com a literatura que relata que homens com vozes normais podem ter maior intensidade de ruído na região superior do espectro ${ }^{1,4}$. Porém, outro estudo encontrou ausência de ruído nas frequências em torno de $3,2 \mathrm{KHz}$ em vozes normais, sendo este achado presente apenas em vozes roucas e ásperas ${ }^{4}$.

Na EBE, houve muita presença de ruído em todo espectro, e presença mediana nas baixas freqüências, sendo que outro estudo levantou a possibilidade de haver ruído em vozes normais ${ }^{19}$ (Tabela 2). O ruído é gerado por vibrações aleatórias decorrentes de fatores aerodinâmicos, neurológicos ou biomecânicos, e não ocorre apenas em vozes patológicas ${ }^{31}$. Portanto, vozes com tom grave e turbulências aéreas podem apresentar ruído, o qual pode ser decorrente, inclusive do fato da fonação ser um processo não linear ${ }^{32-34}$
Ainda, existe relação entre frequência fundamental (f0) grave, vozes roucas e vozes com ruído 4 . Vozes roucas podem apresentar distorção do traçado harmônico (enovelamento), ruído em todo espectro, ruídos adventícios em baixa frequência (mascarando os $\mathrm{h}$ inferiores) e substituição de harmônico por ruído ${ }^{24,25}$. É comum as vozes masculinas apresentarem emissões em registro basal, que produz características espectrográficas semelhantes a quadros funcionais por modelo vocal deficiente ou a presença de massa vibrante, como nos casos de Edema de Reinke ${ }^{1}$. Essas afirmações podem amparar a hipótese de que, pelo fato dos homens apresentarem vozes mais graves, os ciclos glóticos nesse sexo são mais lentos e geram uma voz composta por pulsos glóticos mais espaçados, que podem ser caracterizados como ruído na análise espectrográfica, sem significar, no entanto, que essas vozes sejam disfônicas.

Vozes normais tendem a apresentar um traçado regular e bem definido ${ }^{1,3}$. O traçado irregular ocorre em vozes instáveis, roucas, soprosas e astênicas, sendo que, quebras de sonoridade ou variações de frequência, levam a variações no espectro, como traçado harmônico irregular e presença de espaços falhos $^{16,22,23,26}$. A EBL (Tabela 1) e a EBE (Tabela 2) apresentaram traçados significantemente pouco regulares, achado que pode estar relacionado à instabilidade e tremor vocal por incoordenação pneumofonoarticulatória, comumente encontrada em vozes não treinadas, e pela característica ruidosa dessas vozes, como descrito anteriormente.

A anti-ressonância refere-se ao abafamento acústico decorrente do amortecimento do som. Ocorre quando o som glótico é direcionado para a cavidade nasal, onde os tecidos são mais moles, acarretando perda de energia principalmente nos harmônicos mais superiores². A anti-ressonância na presente pesquisa foi mediana, sendo que em ambas as análises espectrográficas houve significância estatística (Tabelas 1 e 2).

A fraca intensidade no traçado e, em especial nas frequências em torno de $3,2 \mathrm{KHz}$, encontrada no grupo estudado, vai ao encontro de um trabalho que comparou as vozes de cantores treinados com as de falantes não treinados, encontrando nesses últimos redução da intensidade a partir de $\mathrm{F}^{27}$. Numa análise geral, a grande quantidade de ruído nas frequências em torno de $3,2 \mathrm{KHz}^{4,19}$ pode contribuir com a fraca intensidade nessas frequên$\mathrm{Cias}^{27}$, com a pouca definição de $\mathrm{F}^{6,14-16,21-23}$, com a pouca regularidade do traçado ${ }^{16,22,23,26} \mathrm{e} \mathrm{com} \mathrm{a}$ presença mediana de anti-ressonância, que foram também encontrados no grupo estudado. Ainda, a grande quantidade de ruído em todo o espectro ${ }^{4,19}$ e mediana nas baixas ${ }^{4,19}$ pode estar contribuindo 
com a pouca regularidade do traçado, e com a fraca intensidade em todo o espectro ${ }^{27}$.

Os ciclos vibratórios do sinal vocal são semeIhantes, mas não idênticos. Assim, a voz humana é composta por ondas quase periódicas, que fazem com que o sinal vocal não seja perfeito ${ }^{1,14}$ podendo ser considerado por alguns autores como um sistema não-linear, onde estudos baseados na teria do caos vem sendo realizados ${ }^{33-35}$. Pequenas variações e aperiodicidades vocais sempre estarão presentes em sujeitos com laringe e voz normais, e essas variações que são imperceptíveis ao ouvido humano, são detectadas por meio da análise acústica, provando a importância dessa avaliação nos estudos de voz.

As características significantes encontradas neste grupo de homens servem para caracterizar suas vozes espectrograficamente. Esses achados, juntamente com outros estudos, complementam a caracterização de vozes masculinas, como achados recentes que mostraram que existem diferenças entre os sexos quanto a análise dos formantes e da $\mathrm{fO}^{36}$; achados que caracterizam as vogais do português brasileiro e mostram as diferenças acústicas das vogais entre os sexos ${ }^{37}$; e inúmeros achados reunidos e discutidos em revisão de literatura ${ }^{10}$.

\section{CONCLUSÕES}

O grupo de homens adultos jovens, com laringe sem afecções e sem queixas vocais avaliado neste trabalho, mostrou características vocais espectrográficas de muito ruído em todo o espectro e nas frequências em torno de $3,2 \mathrm{KHz}, \mathrm{F} 3$ pouco definido, ruído mediano nas baixas frequências, traçado pouco regular, fraca intensidade em todo espectro e, em especial, no traçado das frequências em torno de $3,2 \mathrm{KHz}$, corroborando a literatura existente sobre vozes masculinas normais. Além da presença de anti-ressonância mediana.

\begin{abstract}
Purpose: to characterize voice spectrography of a group of young adult men. Method: 25 young adult men, without vocal complaints, with otorhinolaryngological larynx diagnosis without disease, have done the supported emission of the vowel [a] standing and with the usual frequency and intensity. The emission was carried thought to analyze Real Time Spectrogram of Kay Pentax ${ }^{\circledR}$, giving a spectrogram of wide band and another of narrow band for each subject. These were doubled in order to increase the reliability of the study and were sent to three judge's language pathologists that have to do the judgment of the same following a protocol. The results were analyzed statically by a test to different from proportion with significant level of $5 \%$. Results: in the wide band spectrograph there was a statistical significance to noise present in the frequencies around $3,2 \mathrm{KHz}$, a little definition of the third formants, a little regularity of the line and anti-resonance average. In the narrow band spectrograph there was weak acutely of the frequencies around $3,2 \mathrm{KHz}$ and in all spectrum, noise is very present throughout the spectrum and in the frequencies around $3,2 \mathrm{KHz}$, noise at low frequencies median, low regularity of the tracing and anti-resonance medium. Conclusion: the spectrographic analyze have been developed in large portion of the noise in all spectrum and in the frequencies around $3,2 \mathrm{KHz}$, little defined third formant, average noise in the low frequencies, the line being a little regular and average anti-resonance, weak acutely in all spectrum and in special, in the frequencies around $3,2 \mathrm{KHz}$.
\end{abstract}

KEYWORDS: Voice; Men's Health; Spectrography; Speech, Language and Hearing Sciences; Larynx

\section{REFERÊNCIAS}

1. Behlau M, Madazio G, Feijó D, Pontes P. Avaliação de Voz. In: Behlau, M. Voz: o livro do especialista vol I. Rio de Janeiro: Revinter, 2001. p.85-245.

2. Pinho SMR, Camargo Z. Introdução à análise acústica da voz e da fala. In: Pinho SMR. Tópicos em Voz. Rio de Janeiro: Guanabara-Koogan; 2001. p. 19-44.

3. Barros APB, Carrara-De Angelis E. Análise acústica da voz. In: Dedivitis RA, Barros APB. Métodos de Avaliação e Diagnóstico da Laringe e Voz. São Paulo: Lovise; 2002. p. 200-21.

4. Pontes PAL, Vieira VP, Gonçalves MIR, Pontes AAL. Características das vozes 
roucas, ásperas e normais: análise acústica espectrográfica comparativa. Revista Brasileira de Otorrinolaringologia. 2002; 68(2): 182-8.

5. González J. Formant frequencies and body size of speaker: a weak relationship in adults humans. Journal of Phonetics. 2004; 32(2): 277-87.

6. Master S, Biase ND, Chiari BM, Pedrosa V. O espectro médio de longo termo na pesquisa e na clínica fonoaudiológica. Pró-Fono Revista de Atualização Científica. 2006; 18(1): 111-20.

7. Xue SA, Hao GJP, Mayo R. Volumetric measurements of vocal tracts for male speakers from different races. Clinical Linguistics \& Phonetics. 2006; 20(9): 691-702.

8. XueSA, Cheng RW, Ng LM. Vocaltract dimensional development of adolescents: An acoustic reflection study. Int J Pediatr Otorhinolaryngol. 2010; 74(8):907-12.

9. Beber BC, Cielo CA. Acoustic measurements of the glottal source of normal male voices. Pró-Fono Revista de Atualização Científica. 2010;22(3):299-304.

10.Beber BC, Cielo CA. Características vocais acústicas de homens com voz e laringe normais.

Rev CEFAC. 2010; 13(2):340-51

11.Barrett KA. Triagem Auditiva de Escolares. In: Katz J. (Org). Tratado de Audiologia Clínica. 4. ed. São Paulo: Manole; 1999. p. 472-85.

12. Schwarz K, Cielo CA. Modificações laríngeas e vocais produzidas pela técnica de vibração sonorizada de língua. Pró-Fono Revista de Atualização Científica. 2009; 21(2):161-6.

13. Oguz $H$, Demirci $M$, Safak MA, Arslan N, Islam A, Kargin s. Effects of unilateral vocal cord paralysis on objective voice measures obtained by Praat. European Archives of Oto-Rhino-Laryngology. 2007; 264(3): 257-62.

14. Russo I, Behlau M. As pistas acústicas das vogais e consoantes. In: Russo I, Behlau M. Percepção da fala: análise acústica do português brasileiro. São Paulo: Lovise; 1993. p. 25-50.

15. Bele IV. The speaker's formant. Journal of Voice. 2006; 20(4):555-78.

16. Leino $\mathrm{T}$, Laukkanen $\mathrm{AM}$, Radolf $\mathrm{V}$. Formation of the actor's/speaker's formant: A study applying spectrum analysis and computer modeling. J Voice. 2010; 25(2):150-8.

17. Côrtes MG, Gama ACC. Visual analysis of spectrographic parameters before and after dysphonia therapy. Rev Soc Bras Fonoaudiol. 2010;15(2):243-9.

18. Zimmer V, Cielo CA, Finger LS. Modificações vocais acústicas espectrográficas produzidas pela fonação reversa. Rev CEFAC. 2010; 12(4):535-42.

19. Valentim AF, Côrtes MG, Gama ACC. Spectrographic analysis of the voice: effect of visual training on the reliability of evaluation. Rev Soc Bras Fonoaudiol. 2010; 15(3):335-42.

20. Souza EMG. Para a caracterização fonéticoacústica da nasalidade no português do Brasil. Dissertação (Mestrado) - Instituto de Estudos da Linguagem Universidade Estadual de Campinas, Campinas, 1994.

21. Bjorkner E. Musical theater and opera singingWhy so different? A study of subglottal pressure, voice source, and formant frequency characteristics. Journal of Voice. 2008; 22(5): p. 533-40.

22. Howard DM. Acoustics of the trained versus untrained singing voice. Current Opinion in Otolaryngology \& Head and Neck Surgery. 2009; 17:155-9.

23. Ferguson S, Kenny DT, Cabrera D. Effects of training on time-varying spectral energy and sound pressure level in nine male classical singers. Journal of Voice. 2010; 24(1): 39-46.

24. Hirano M. Clinical applications of voice tests assesment of speech and voice production: research and clinical application NICD monograph preceedings of a conference of the National Institute of Health NICD. Maryland; 1990. p. 196-203.

25. Hammarberg B, Gauffin J. Perceptual and acoustical characteristics of quality differences in pathological voices as related to physiological aspects. In: Fujimura O, Hirano M. Vocal Fold Physiology, Voice Quality Control. San Diego: Singular, San Diego; 1995. p. 283-303.

26. Batalla, F.N.; Santos, P.C.; Santiago, G.S.; González, B.S.; Nieto, C.S. Evaluación espectral cuantitativa de la hipofunción vocal. Acta Otorrinolaringol Esp. 2004; 55: 327-33.

27. Barrichelo VMO, Heuer RJ, Dean CM, Sataloff, RT. Comparison of singer's formant, speaker's ring, and LTA spectrum among classical singers and untrained normal speakers. J Voice. 2001; 3:344-50. 28. Yanagihara, N. Significance of harmonic changes and noise components in hoarseness. Journal of Speech and Hearing Research. 1967; 30: 431-541.

29. Iglesia FV, González SF, Gómez MC. Evaluación espectral cualitativa de la voz esofágica. Acta Otorrinolaringol Esp. 2006; 57: 319-23.

30. Martens JWMAF, Versnel H, Dejonckere PH. The effect of visible speech in the perceptual rating of pathological voices. Arch Otolaryngol Head Neck Sur. 2007; 133(2): 178-85.

31. Jiang JJ, Zhang Y. Chaotic vibration induced by turbulent noise in a two-mass model of vocal folds. J Acoust Soc Am. 2002; 112(5): 2127-33.

32. Batalla, F.N.; Santos, P.C.; Santiago, G.S.; González, B.S.; Nieto, C.S. Evaluación perceptual de la disfonia: correlación con los parâmetros acústicos y fiabilidad. Acta Otorrinolaringol Esp. 2004; 55: 282-7. 
33. Tao C, Jiang JJ. Effects of mucosal loading on vocal fold vibration. Chaos. 2009; 19(2): 23-113.

34. Jiang JJ, Zhang Y, McGilligan C. Chaos in voice, from modeling to measurement. $\mathrm{J}$ Voice. 2006; 20(1):2-17.

35. Tao C, Jiang JJ. Chaotic component obscured by strong periodicity in voice production system. Phys Rev E Stat Nonlin Soft Matter Phys. 2008; 77(6 Pt 1):619-22.
36. Hillenbrand JM, Michael JC. The role of $f 0$ and formant frequencies in distinguishing the voices of men and women. Attention, Perception, \& Psychophysics. 2009; 71(5):1150-66.

37. Gonçalves MIR, Pontes PAL, Vieira VP, Pontes AAL, Curcio D, Biase NG. Função de transferência das vogais orais do Português brasileiro: análise acústica comparativa. Braz J Otorhinolaryngol. 2009;75(5):680-4.

http://dx.doi.org/10.1590/S1516-18462012005000008

RECEBIDO EM: 28/09/2010

ACEITO EM: 03/09/2011

Endereço para correspondência:

Barbara Costa Beber

Rua Juvenal Custódio Oliveira, 318

Bairro Navegantes - Palmares do Sul - RS

CEP: 95540-000

E-mail: bcbfono@yahoo.com.br 